\title{
VARIAÇÃO SAZONAL DA COMPOSIÇÃO DO ÓLEO ESSENCIAL DE Myrcia salzmannii Berg. (Myrtaceae)
}

\author{
Martins Dias de Cerqueira, Edson de Jesus Marques, Dirceu Martins, Nídia F. Roque e Frederico Guaré Cruz* \\ Instituto de Química, Universidade Federal da Bahia, Campus de Ondina, 40170-290 Salvador - BA, Brasil \\ Maria Lenise da Silva Guedes \\ Instituto de Biologia, Universidade Federal da Bahia, Campus de Ondina, 40170-290 Salvador - BA, Brasil
}

Recebido em 19/8/08; aceito em 28/1/09; publicado na web em 3/7/09

\begin{abstract}
SEASONAL VARIATION OF THE COMPOSITION OF ESSENTIAL OIL FROM Myrcia salzmannii Berg. (Myrtaceae). This work report the seasonal variation of composition of the volatile oils from leaves and from flowers of Myrcia salzmannii harvested in the sand dunes of Salvador, Bahia, northeastern region of Brazil in the years 2001 and 2003. The oils were analyzed by GC-FID and GC-MS being identified 49 components. Nine essential oil samples of leaves collected on different months and years and one sample of flowers were analyzed. $\beta$-Caryophyllene and $\alpha$-humulene were the only compounds present in all of the samples being the first the majority compound.
\end{abstract}

Keywords: Myrcia salzmannii; essential oil; terpenes.

\section{INTRODUÇÃO}

As plantas aromáticas têm sido utilizadas desde a Antigüidade como antissépticos e agentes anti-infecciosos, para dar aroma a perfumes e cosméticos e como flavorizantes em alimentos e bebidas. Suas propriedades biológicas estão diretamente relacionadas com a composição química, a qual pode ser afetada pelas variações ambiental, geográfica, sazonal e circadiana.

As espécies da família Myrtaceae são particularmente ricas em óleos essenciais. Essa família é constituída de 140 gêneros e aproximadamente 3000 espécies $^{1}$ com dois principais centros de desenvolvimento, a América tropical e a Austrália, embora ocorram em outras regiões no mundo. ${ }^{2} \mathrm{O}$ gênero Myrcia é um dos maiores da família com cerca de 300 espécies e muito bem representado em todo o território brasileiro. Espécies desse gênero têm sido utilizadas na medicina popular como adstringentes, diuréticos, contra o Diabetes mellitus, para estancar hemorragias e no tratamento de hipertensão e úlceras. ${ }^{3}$

Relativamente ao seu grande número, são poucas as publicações acerca da química das espécies do gênero Myrcia. Em M. citriofolia, foi relatada a ocorrência de eucaliptina, uma flavona $\mathrm{C}$-metilada. ${ }^{4} \mathrm{O}$ extrato metanólico de $M$. multiflora mostrou potente atividade inibitória da aldose redutase, ${ }^{5,6}$ uma enzima que está relacionada com algumas complicações do Diabetes mellitus. ${ }^{7,8} \mathrm{O}$ fracionamento desse extrato levou ao isolamento de flavanonas glicosiladas (myrciacitrinas I e II) e acetofenonas glicosiladas (myrciafenonas A e B). Os óleos essenciais de 17 espécies do gênero já foram estudados e ficou demonstrado que os sesquiterpenos foram predominantes em todos eles exceto em $M$. myrtifolia,${ }^{9}$ em que houve uma larga predominância de monoterpenos e $M$. acuminatissima e M. bombycina, nos quais o conteúdo de monoterpenos foi apenas ligeiramente maior que o de sesquiterpenos. ${ }^{10-12}$

Neste trabalho descrevemos a variação na composição química dos óleos essenciais extraídos das folhas de $M$. salzmannii coletadas em diferentes meses dos anos de 2001 e 2003. Adicionalmente, relatamos a composição química do óleo essencial extraído das flores coletadas em fevereiro de 2003.

\footnotetext{
*e-mail: fguare@ufba.br
}

\section{PARTE EXPERIMENTAL}

\section{Material vegetal}

As coletas foram realizadas em diferentes meses dos anos de 2001 e 2003 em dois espécimes de M. salzmannii presentes no Parque Metropolitano da Lagoa do Abaeté, Salvador, BA. As flores foram coletadas de um dos espécimes no mês de fevereiro de 2003. As folhas e flores depois de coletadas foram cuidadosamente separadas e acondicionadas sob refrigeração $\left(-20^{\circ} \mathrm{C}\right)$ até a extração. Uma exsicata está depositada no Herbário Alexandra Leal Costa, no Instituto de Biologia da Universidade Federal da Bahia (UFBA), sob o número ALCB - 04761 .

\section{Análise dos óleos essenciais}

Os óleos essenciais foram obtidos por hidrodestilação com uma aparelhagem tipo Clevenger adaptada, por um período de ebulição de $3 \mathrm{~h}$. As análises por cromatografia a gás foram feitas em um cromatógrafo HP 5890, equipado com uma coluna capilar DB-5 (30 m x $0,25 \mathrm{~mm}$ e filme de $0,25 \mu \mathrm{m}$ de espessura) e detector de ionização de chama. $\mathrm{O}$ gás de arraste utilizado foi o hidrogênio com fluxo de 1 $\mathrm{mL} / \mathrm{min}$. A temperatura de trabalho do detector foi de $300{ }^{\circ} \mathrm{C}$, sendo a mistura de gases de queima constituída de hidrogênio, com fluxo de $30 \mathrm{~mL} / \mathrm{min}$, nitrogênio, com fluxo de $30 \mathrm{~mL} / \mathrm{min}$ e ar sintético, com fluxo de $300 \mathrm{~mL} / \mathrm{min}$. A temperatura do injetor foi mantida em $220{ }^{\circ} \mathrm{C}$ durante toda a análise. A temperatura do forno foi programada para iniciar com $60^{\circ} \mathrm{C}$ e aumentar a uma taxa de $3{ }^{\circ} \mathrm{C} / \mathrm{min}$ até a temperatura de $240^{\circ} \mathrm{C}$ e manter-se por $10 \mathrm{~min}$ nesta temperatura. Os cromatogramas de íons totais e os espectros de massas das substâncias contidas nos óleos essenciais foram obtidos utilizando-se um cromatógrafo a gás HP 6890 equipado com uma coluna capilar HP-5MS (30 m x 0,25 mm e filme de $0,25 \mu \mathrm{m}$ de espessura), interfaciado com um detector seletivo de massas HP 5973 operando com energia de ionização de 70 eV. O gás de arraste utilizado foi o hélio, com uma vazão de $1 \mathrm{~mL} /$ min, o volume de injeção foi de $1 \mu \mathrm{L}$ de solução do óleo em diclorometano com uma taxa de divisor de fluxo de 1:100. A programação de temperatura do forno foi a mesma utilizada nas análises por CG-DIC, sendo a temperatura do injetor mantida $200{ }^{\circ} \mathrm{C}$. 
As substâncias foram identificadas por comparação dos seus espectros de massas com os espectros da base de dados NIST 98 (NIST Mass Spectral Library, 1998) e os registrados na literatura ${ }^{13,14}$ e por comparação de seus índices de retenção, obtidos a partir do algoritmo de Kovats modificado ${ }^{15,16}$ utilizando como padrões uma série homóloga de hidrocarbonetos lineares de $\mathrm{C}_{9} \mathrm{H}_{20}$ (nonano) a $\mathrm{C}_{26} \mathrm{H}_{54}$ (hexacosano), com os índices de retenção registrados na literatura. ${ }^{13,14}$

\section{RESULTADOS E DISCUSSÃO}

A região de Salvador, BA, pode ser caracterizada, basicamente, por duas estações climáticas: o "verão", com temperaturas variando entre 26 e $34{ }^{\circ} \mathrm{C}$, no qual ocorrem apenas chuvas ocasionais não prolongadas com início em meados de setembro estendendo-se até o final de fevereiro, e o "inverno", com temperaturas variando entre 20 e $28{ }^{\circ} \mathrm{C}$, com longos e frequentes períodos chuvosos, iniciando-se em março e estendendo-se, às vezes, até setembro.

Para verificar a influência do clima na composição do óleo essencial (OE) de $M$. salzmannii foram realizadas análises do OE das folhas coletadas no final de fevereiro de 2001, final do "verão" e do OE das folhas coletadas no início de outubro de 2001, final do "inverno". As composições dos dois óleos foram qualitativa e quantitativamente diferentes (Tabela 1). No OE de fevereiro de 2001 foram detectados 15 componentes, dos quais 13 foram identificados, já no OE de outubro de 2001 foram detectados 9 componentes e foram identificados 6 . Nos dois casos o principal constituinte foi o $\beta$-cariofileno que em fevereiro de 2001 estava presente na concentração de $41,5 \%$ e em outubro de 2001 na concentração de $24,1 \%$. Onze dos 15 compostos detectados em fevereiro de 2001 não estavam presentes no OE de outubro do mesmo ano. Dentre eles destacam-se o eucaliptol, o germacreno D, o $\alpha$-cadineno, o selina-3,7(11)-dieno, o $\alpha$-calacoreno, o álcool cariofilênico e o óxido de cariofileno, todos com concentrações maiores do que $2 \%$. No OE de outubro de 2001 , três dos seis componentes identificados, o $\alpha$-pineno, o terpineol e o $\alpha$-copaeno não estavam presentes no OE de fevereiro. Deve-se ainda destacar que o $\alpha$-humuleno, o segundo maior componente do OE de outubro com 10,8\%, estava presente com apenas 3,8\% no OE de fevereiro. Nas duas amostras de óleo os sesquiterpenos foram largamente predominantes, porém, no OE de fevereiro de 2001 a quantidade de sesquiterpenos foi muito maior do que no OE de outubro de 2001.

Estes resultados sugeriram que a diferença na composição dos OE poderia ser atribuída às variações climáticas próprias das duas estações. Para verificar essa hipótese foram realizadas análises com amostras coletadas bimestralmente a partir de fevereiro até dezembro de 2003 (Tabela 1)

A comparação da composição dos $\mathrm{OE}$ de fevereiro e outubro de 2001 com os de fevereiro e outubro de 2003, respectivamente, mostra que os $\mathrm{OE}$ são qualitativa e quantitativamente diferentes. Oito componentes, com mais de $2 \%$, presentes em fevereiro de 2001 não estavam presentes em fevereiro de 2003 e seis componentes presentes nesta amostra de óleo não estavam presentes em fevereiro de 2001. Já as composições dos OE de outubro foram, aproximadamente, semelhantes.

De maneira geral pôde-se verificar que existe uma tendência de o $\alpha$-pineno se apresentar em maiores concentrações de abril a dezembro, estando ausente em fevereiro. $\mathrm{O} \beta$-cariofileno foi sempre o componente em maior concentração apresentando concentração máxima em fevereiro de 2001 e mínima em abril de 2003. Outro componente marcante nos $\mathrm{OE}$ foi o $\alpha$-humuleno. Excetuando-se os meses de fevereiro de 2001 e abril de 2003 sua concentração foi relativamente alta e entre 10,3 e 13,2\% (Figura 1). Em geral, as espécies do gênero Myrcia, na Lagoa do Abaeté, florescem de outubro a dezembro e as flores permanecem por dois ou três dias. Anormalmente, nós encontramos um espécime florido em fevereiro de 2003. No OE das flores foram detectados vinte e oito componentes (Tabela 1) dos quais vinte foram identificados. Cinco componentes destacaram-se pela sua maior concentração: o $\beta$-cariofileno, o mais abundante deles com $(13,8 \%)$, o $\alpha$-humuleno $(10,9 \%)$ e três outros não identificados com índices de retenção de1582 (10,0\%), 1586 $(12,6 \%)$ e $1602(7,1 \%)$.

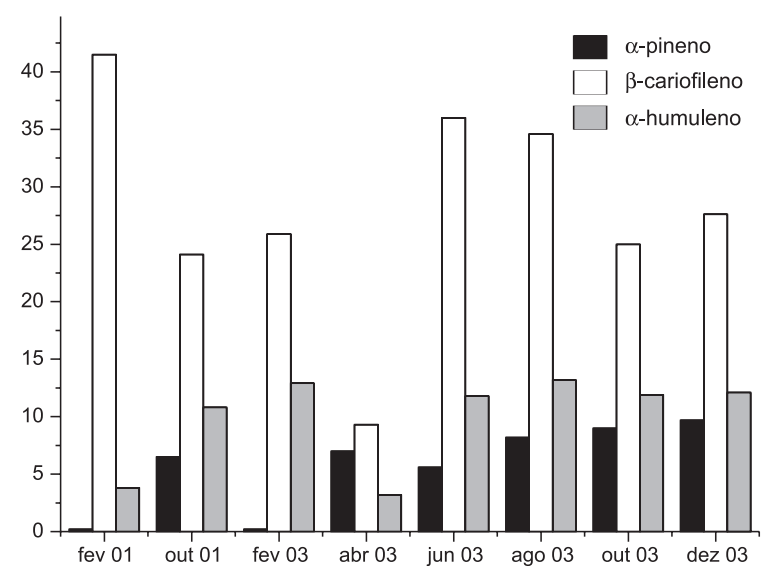

Figura 1. Variação percentual dos principais componentes do óleo essencial obtido das folhas de Myrcia salzmannii em diferentes coletas

Pelo fato da espécie M. salzmannii apresentar-se como pequenos arbustos no local de coleta, a quantidade de material (folhas e flores) coletada foi sempre pequena, ao redor de $100 \mathrm{~g}$. Com isso, não foram obtidas quantidades de OE suficientes para se avaliar o rendimento com os métodos disponíveis.

\section{CONCLUSÃO}

Foram identificados 49 compostos sendo o $\beta$-cariofileno o componente majoritário em todas as amostras (Tabela 1). Em relação à concentração dos componentes majoritários, excetuandose o OE de fevereiro de 2001 e o OE das flores, a concentração do $\beta$-cariofileno foi sempre de duas a três vezes a concentração do $\alpha$-humuleno. Houve uma predominância de sesquiterpenos em todas as amostras, o que está de acordo com os resultados obtidos anteriormente para a maioria das espécies de Myrcia. ${ }^{10-12}$ Um exame da Tabela 1 indica que houve variações qualitativas e quantitativas na composição do óleo essencial ao longo dos meses de 2003. No entanto, quando se consideram as médias das concentrações de abril, junho e agosto, meses de "inverno", e as médias de outubro, dezembro e fevereiro, meses de "verão", do ano de 2003, observam-se valores próximos de concentração para os três componentes principais identificados: $\alpha$-pineno, 6,9 e $6,2 \%$, cariofileno, 26,3 e $26,1 \%$, $\alpha$-humuleno, 9,4 e 12,3\%, respectivamente. Porém, outros componentes como os não identificados (NI) de maior concentração: NI com IK de 1582, NI com IK de 1586 e NI com IK de 1602 tiveram a média de suas concentrações nos meses de verão aproximadamente o dobro da média de suas concentrações no inverno. É possível, portanto, inferir que o regime de chuvas pode influir na concentração de alguns componentes e não na de outros. No entanto, as variações observadas nos resultados obtidos para cada amostra são indicativos de que uma rede complexa de fatores e/ou condições ambientais, tais como temperatura, umidade, duração e intensidade das irradiações solares, interações com polinizadores e predadores, ${ }^{17-19}$ estão influenciando a composição do óleo e não apenas do regime de chuvas. 


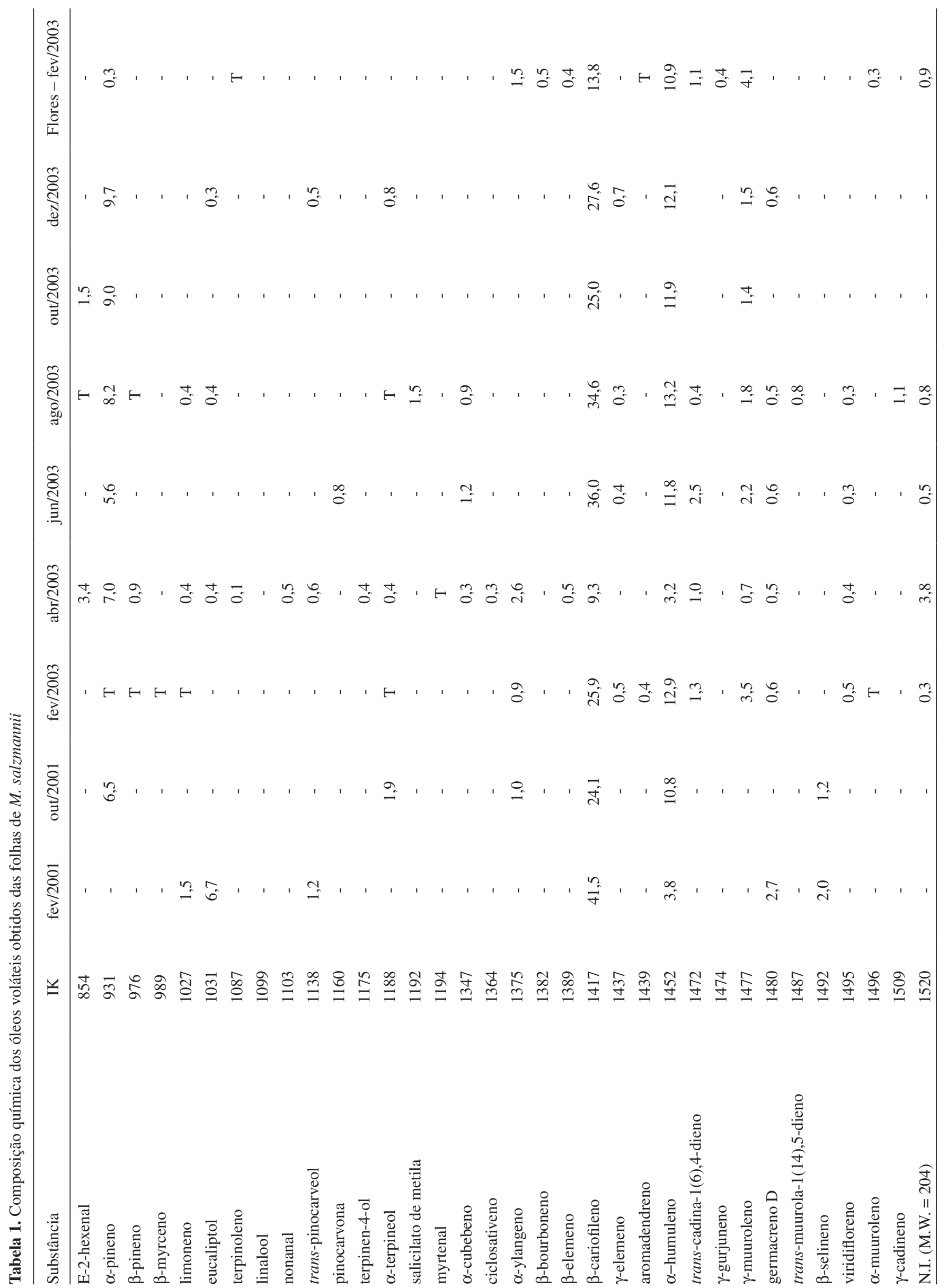




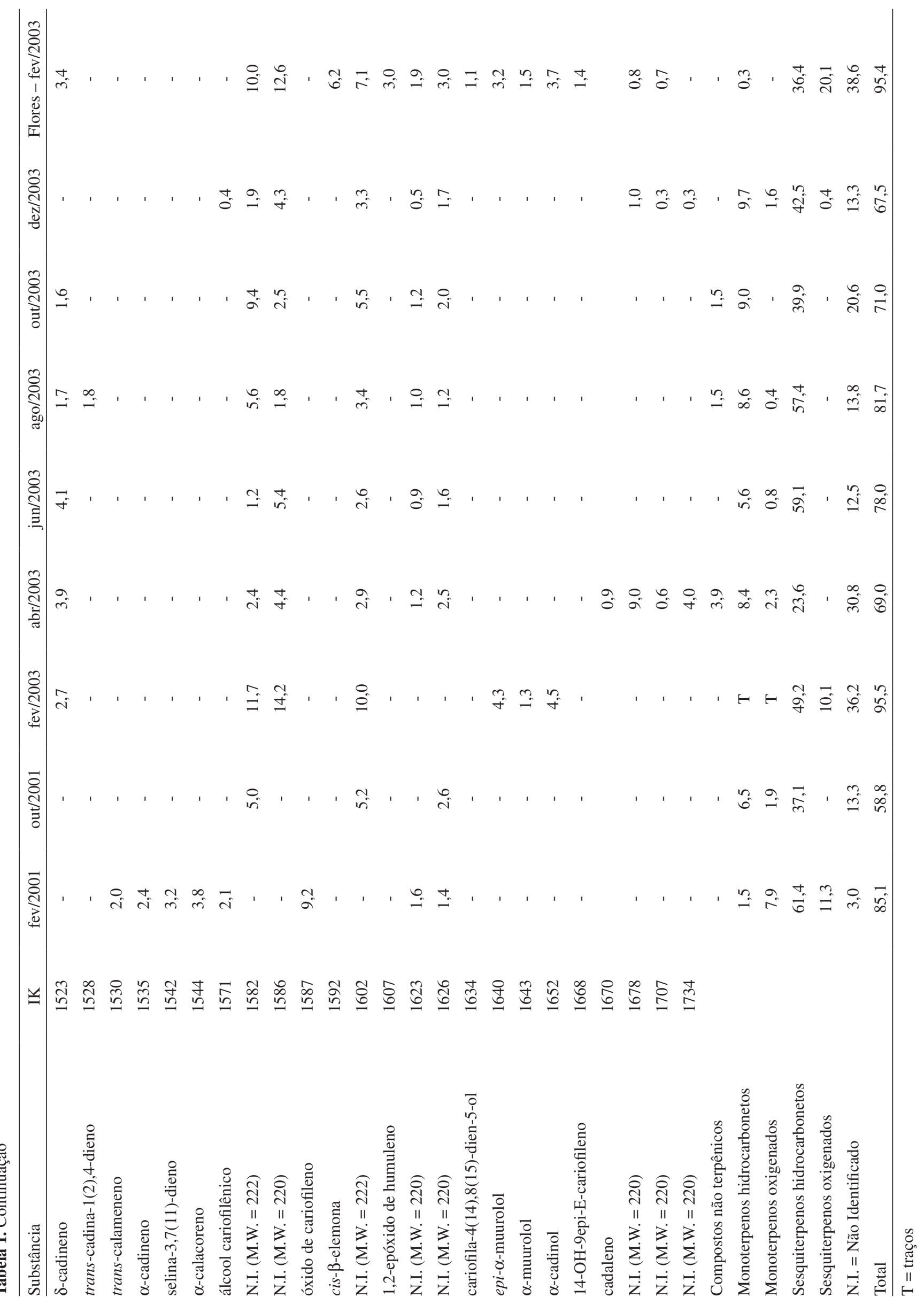




\section{AGRADECIMENTOS}

Ao CNPq, CAPES, FINEP e FAPESB pelos auxílios e bolsas concedidas.

\section{REFERÊNCIAS}

1. Ribeiro, J. E. L. da S.; Hopkins, M. J. G.; Vicentini, A.; Sothers, C. A.; Costa, M. A. Da S.; de Brito, J. M.; de Souza, M. A. D.; Martins, L. H. P.; Lohmann, L. G.; Assunção, P. A. C. L.; Pereira, E. da C.; da Silva, C. F.; Mesquita, M. R.; Procópio, L. C.; Flora da Reserva Ducke - Guia de Identificação das plantas vasculares de uma floresta de terra firme na Amazônia Central, INPA: Manaus, 1999.

2. Joly, A. B.; Introdução à taxonomia vegetal, CEN: São Paulo, 1979.

3. Russo, E. M. K.; Reichelt, A. A. J.; Desa, J. R.; Furlanetto, R. P.; Moises, R. C. S.; Kasamatsu, T. S.; Chacra, A. R.; Braz. J. Med. Biol. Res. 1990, $23,11$.

4. Gottilieb, O. R.; da Silva, M. L.; Maia, J. G. S.; Phytochemistry 1972, 11,1185 .

5. Yoshikawa, M.; Shimada, H.; Nishida, N.; Li, Y.; Toguchida, I.; Yamahara, J.; Matsuda, H.; Chem. Pharm. Bull. 1998, 46, 113.

6. Matsuda, H.; Nishida, N.; Yoshikawa, M.; Chem. Pharm. Bull. 2002, 50, 429.

7. Srivastava, S. K.; Ramana, K. V.; Chandra, D.; Srivastava, S.; Bhatnagar, A.; Chem. Biol. Interact. 2003, 143, 333.
8. Robison, W. G.; Lavert, N. M.; Lou, M. F.; Progress in Retinal and Eye Research 1995, 14, 593.

9. De Cerqueira, M. D.; Souza-Neta, L. C.; Passos, M. G. V. M.; Lima, E. O.; Roque, N. F.; Martins, D.; Guedes, M. L. S.; Cruz, F. G.; J. Braz. Chem. Soc. 2007, 18, 998.

10. Henriques, A. T.; Sobral, M.; Bridi, R.; Vérin, P.; Menut, C.; Lamaty, G.; Bessière, J. M.; J. Essent. Oil Res. 1997, 9, 13.

11. Zoghbi, M. D.; Andrade, E. H. A.; da Silva, M. H. L.; Carreira, L. M. M.; Maia, J. G. S.; Flavour Frag. J. 2003, 18, 421.

12. Limberger, R. P.; Sobral, M.; Henriques, A. T.; Menut, C.; Bressière, J. M.; Quim. Nova 2004, 27, 916.

13. Adams, R. P.; Identification of Essencial Oil Components by Gas Chromatography/Quadrupole Mass Spectroscopy, Allured Publishing Corp: Carol Stream, IL, USA, 2001.

14. Joulain, D.; Köning, W. A.; The atlas of spectral data of sesquiterpene hydrocarbons, E. B. - Verlag: Hamburg, 1998.

15. Kovats, E.; Helv. Chim. Acta 1958, 41, 1915.

16. Van den Dool, H.; Kratz, P. D.; J. Chromatogr. 1963, 11, 463.

17. Sangwan, N. S.; Farooqi, A. H. A.; Shabih, F.; Sangwan, R. S.; Plant Growth Regulation 2001, 34, 3.

18. Lopes, N. P.; Kato, M. J.; Andrade, E. H. A.; Maia, J. G. S.; Yoshida, M.; Phytochemistry 1997, 46, 689.

19. Carvalho Filho, J. L. S.; Blank, A. F.; Alves, P. B.; Ehlert, P. A. D.; Melo, A. S.; Sócrates C. H. Cavalcanti, S. C. H.; Arrigoni-Blank, M. F.; SilvaMann, R.; Rev. Bras. Farmacog. 2006, 16, 24. 